\title{
Birth weight in relation to health and disease in later life: an umbrella review of systematic reviews and meta-analyses
}

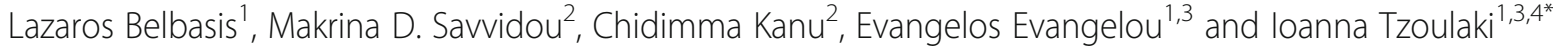

\begin{abstract}
Background: Birth weight, a marker of the intrauterine environment, has been extensively studied in epidemiological research in relation to subsequent health and disease. Although numerous meta-analyses have been published examining the association between birth weight and subsequent health-related outcomes, the epidemiological credibility of these associations has not been thoroughly assessed. The objective of this study is to map the diverse health outcomes associated with birth weight and evaluate the credibility and presence of biases in the reported associations.
\end{abstract}

Methods: An umbrella review was performed to identify systematic reviews and meta-analyses of observational studies investigating the association between birth weight and subsequent health outcomes and traits. For each association, we estimated the summary effect size by random-effects and fixed-effects models, the $95 \%$ confidence interval, and the $95 \%$ prediction interval. We also assessed the between-study heterogeneity, evidence for smallstudy effects and excess significance bias. We further applied standardized methodological criteria to evaluate the epidemiological credibility of the statistically significant associations.

Results: Thirty-nine articles including 78 associations between birth weight and diverse outcomes met the eligibility criteria. A wide range of health outcomes has been studied, ranging from anthropometry and metabolic diseases, cardiovascular diseases and cardiovascular risk factors, various cancers, respiratory diseases and allergies, musculoskeletal traits and perinatal outcomes. Forty-seven of 78 associations presented a nominally significant summary effect and 21 associations remained statistically significant at $P<1 \times 10^{-6}$. Thirty associations presented large or very large betweenstudy heterogeneity. Evidence for small-study effects and excess significance bias was present in 13 and 16 associations, respectively. One association with low birth weight (increased risk for all-cause mortality), two dose-response associations with birth weight (higher bone mineral concentration in hip and lower risk for mortality from cardiovascular diseases per $1 \mathrm{~kg}$ increase in birth weight) and one association with small-for-gestational age infants with normal birth weight (increased risk for childhood stunting) presented convincing evidence. Eleven additional associations had highly suggestive evidence.

Conclusions: The range of outcomes convincingly associated with birth weight might be narrower than originally described under the "fetal origin hypothesis" of disease. There is weak evidence that birth weight constitutes an effective public health intervention marker.

Keywords: Bias, Birth weight, Epidemiological credibility, Meta-analysis

\footnotetext{
* Correspondence: i.tzoulaki@imperial.ac.uk

${ }^{1}$ Department of Hygiene and Epidemiology, University of loannina Medical

School, loannina, Greece

${ }^{3}$ Department of Epidemiology and Biostatistics, School of Public Health,

Imperial College London, London, UK

Full list of author information is available at the end of the article
} 


\section{Background}

In early 1990's, the "fetal origin hypothesis" of adult diseases was suggested to describe the observed associations between low birth weight (BW) and cardiovascular diseases in adult life [1-5]. Barker, who first observed these associations, hypothesized that fetal under-nutrition may lead to disproportionate fetal growth and program later coronary heart disease risk [6].

Since then, the importance of the early life and intrauterine environment in relation to later disease has been widely acknowledged and studied $[1,6-10]$. BW is considered a marker of the intrauterine environment and has been extensively studied in epidemiological research, both in terms of its predictors but mainly in relation to subsequent disease. The examined phenotypes expanded beyond cardiovascular conditions into a wide range of outcomes and traits, including respiratory disease $[8,11]$, cancer $[12,13]$ and psychiatric outcomes [14]. At the same time, acknowledging its importance, WHO included low BW ( $<2500 \mathrm{~g})$ as one of its 2025 targets, namely a $30 \%$ reduction in the number of infants born with a BW below $2500 \mathrm{~g}$ by 2025 [15]. During the last two decades, interest in the potential health risks associated with high BW (>4000 g) has also emerged, and associations between high BW and the risk of adverse health outcomes have been studied in an increasing number of scientific papers.

Interpreting associations between $\mathrm{BW}$ and the occurrence of health problems later in life is, however, challenging and linked to a series of methodological limitations [16]. Despite the attention that BW has received in public health policy and epidemiological research, a comprehensive assessment of the proposed associations between BW and future disease is lacking. In the current study, we applied the methodology of umbrella reviews to map all the outcomes that have been associated with low and high BW and we applied a standardized approach to assess the credibility of the findings in order to identify which associations are supported by robust evidence.

\section{Methods}

\section{Literature search and eligibility criteria}

We performed an umbrella review, which is a comprehensive and systematic collection and evaluation of multiple systematic reviews and meta-analyses performed on a specific research topic [17]. We followed a standardized procedure that has already been applied in the appraisal of observational associations in other research fields [18-21]. We systematically searched PubMed from inception to December 24, 2015, to identify systematic reviews and meta-analyses of observational studies examining associations of BW with medical conditions, traits and biomarkers. We used the following search algorithm: ("birth weight" OR "birth size" OR "small for gestational age" OR "large for gestational age" OR "fetal growth restriction" OR "intra-uterine growth restriction") AND (systematic review* OR systematic literature review* OR meta-analys*). We excluded meta-analyses examining genetic or environmental determinants of BW. We further excluded the meta-analyses of individual participant data that did not report the study-specific estimates and pooled analyses that only summarized evidence across a nonsystematically selected number of cohort studies or that did not present the study-specific effect estimates of component studies [22-27]. We did not apply any limitation based on language of publication.

\section{Data extraction}

Two independent researchers extracted the data (LB, CK), and in the case of discrepancies, the final decision was that of a third researcher (IT). From each eligible article, we recorded the first author, journal, year of publication, examined outcomes and number of studies included. We also extracted the study-specific effect sizes (risk ratio, odds ratio, hazard ratio, mean difference and regression coefficient) along with the corresponding $95 \%$ confidence intervals and the number of cases and controls in each study for each association. Whenever the sample sizes were not available through the metaanalysis, we retrieved the original reports to record them. Further, when multiple comparisons were available for a particular phenotype (e.g. < 2500 g vs. $\geq$ $2500 \mathrm{~g}$ and $<2500 \mathrm{~g}$ vs. $2500-4000 \mathrm{~g})$ we always preferred to extract information on $<2500 \mathrm{~g}$ versus $\geq 2500 \mathrm{~g}$ and $>4000 \mathrm{~g}$ versus $\leq 4000 \mathrm{~g}$ in the case of low BW and high $\mathrm{BW}$, respectively. However, when this comparison was not available, we extracted the comparison reported by the meta-analysis. For the excluded meta-analyses assessing an overlapping association, we recorded the level of comparison and the summary effect estimate along with the $95 \%$ confidence interval. Additionally, we scrutinized the full-text of the eligible papers to examine whether their authors discussed the potential effect of gestational age in the association of BW with subsequent health outcomes.

\section{Statistical analysis}

For each meta-analysis, we estimated the summary effect size and its $95 \%$ confidence interval with both fixedeffects and random-effects models $[28,29]$. We also estimated the $95 \%$ prediction interval, which further accounts for between-study heterogeneity and evaluates the uncertainty for the effect that would be expected in a new study addressing that same association [30, 31].

In the case of meta-analyses with continuous outcomes, the standardized mean difference was transformed to an odds ratio with an established formula [32]. Between-study heterogeneity was assessed by the $\mathrm{I}^{2}$ metric [33]. $\mathrm{I}^{2}$ ranges between $0 \%$ and $100 \%$ and is the 
ratio of between-study variance over the sum of the within-study and between-study variances [34]. Values exceeding $50 \%$ or $75 \%$ are usually judged to represent large or very large heterogeneity, respectively.

We assessed whether there was evidence for smallstudy effects (i.e. whether smaller studies tend to give substantially larger estimates of effect size compared with larger studies) with the regression asymmetry test proposed by Egger et al. [35, 36]. A $P$ value less than 0.10 with a more conservative effect in the largest study than in random-effects meta-analysis was judged to be evidence for small-study effects.

We applied the excess statistical significance test, which assesses whether the observed number of studies with nominally significant results is larger than their expected number [37]. This test assesses whether the number of positive studies among those in a metaanalysis is too large based on the power that these studies have to detect plausible effects at an $\alpha$ of 0.05 . The expected number of studies with significant results is calculated in each meta-analysis by the sum of the statistical power estimates for each component study. The power of each component study was estimated using the effect size of the largest study (smallest SE) in a meta-analysis and the power calculation was based on an algorithm using a non-central $t$ distribution [38, 39]. Excess statistical significance for single meta-analyses was claimed at $P<0.10$ [37]. For four associations, the power calculations and the excess statistical significance test were not performed, because the sample sizes of the component studies could not be retrieved neither from meta-analysis papers nor from the original reports.

Finally, we identified the associations that had the strongest validity and were not suggestive of bias. Specifically, we considered as convincing the associations that met the following criteria: significance under the random-effects model at $P<1 \times 10^{-6}$, more than 1000 cases, not large between-study heterogeneity $\left(\mathrm{I}^{2}<\right.$ $50 \%), 95 \%$ prediction interval excluding the null value, and no evidence of small-study effects and excess significance bias. Additionally, the associations with a statistically significant effect at $P<1 \times 10^{-6}$, more than 1000 cases, and a statistically significant effect in the largest study were characterized as having highly suggestive evidence. We considered as suggestive the associations that have more than 1000 cases and a statistically significant effect under the random-effects model at $P<1 \times 10^{-3}$. The rest of statistically significant associations at $P<0.05$ under random-effects model were graded as weak associations.

The statistical analyses were performed with STATA version 12.0 and the power calculations were performed using STATA version 12.0 and G*Power version 3.1.

\section{Results}

Overall, the literature search identified 1520 articles, of which 39 articles, published between 2005 and 2015, were deemed eligible (Fig. 1). Sixty-three papers were screened by full-text. Of these, 10 examined the same or related phenotypes in the same population (defined as overlapping meta-analysis), six were individual participant data meta-analyses that did not report the studyspecific effect estimates, and 12 were systematic reviews without a quantitative synthesis. The 39 eligible papers included 78 different meta-analyses (Table 1): 28 assessing low BW, four assessing small-for-gestational age infants, 18 assessing high $\mathrm{BW}$, and 28 assessing a doseresponse association between $\mathrm{BW}$ and subsequent health outcomes. A wide range of health outcomes has been studied ranging from anthropometry and metabolic disease, cardiovascular disease and cardiovascular risk factors, various cancers, respiratory diseases and allergies, musculoskeletal traits, and perinatal outcomes. Both neonatal and childhood conditions as well as adult diseases have been extensively examined (Table 1). Only two eligible papers had access to raw data of primary studies and performed an individual-level data metaanalysis $[8,40]$.

Overall, we identified more than one published metaanalysis for 25 outcomes, i.e. meta-analysis examining the same exposure (birth weight) and the same outcome. Overlapping meta-analyses provided concordant results, with the exception of two pairs, which had a summary effect in opposite direction (diastolic blood pressure and overweight) compared to the meta-analysis included in our umbrella review (largest most recently published meta-analysis). Six other meta-analyses differed in the summary effect significance compared to the most recent one (Additional file 1: Table S1).

\section{Associations with low BW}

Across 28 meta-analyses examining low BW as a dichotomous trait, the median number of cases was 5766 (interquartile range (IQR), 1574-11,729), while the median number of datasets was 11 (IQR, 8-16). Overall, 21 out of 28 associations had more than 1000 cases, 17 of 28 meta-analyses presented a nominally significant effect $(P<0.05)$ and 10 of them had a significant effect at $P<$ 0.001 . Only seven meta-analyses, examining the association of low BW with perinatal mortality in developing countries, wheezing disorders in childhood, being overweight or obese in adulthood, coronary heart disease, intelligence in adolescence, all-cause mortality, and chronic kidney disease, were statistically significant at $P$ $<1 \times 10^{-6}$ under the random-effects model (Table 1). The largest study had a standard error of less than 0.10 in 17 meta-analyses and a more conservative effect compared to random-effects model in 15 meta-analyses. 


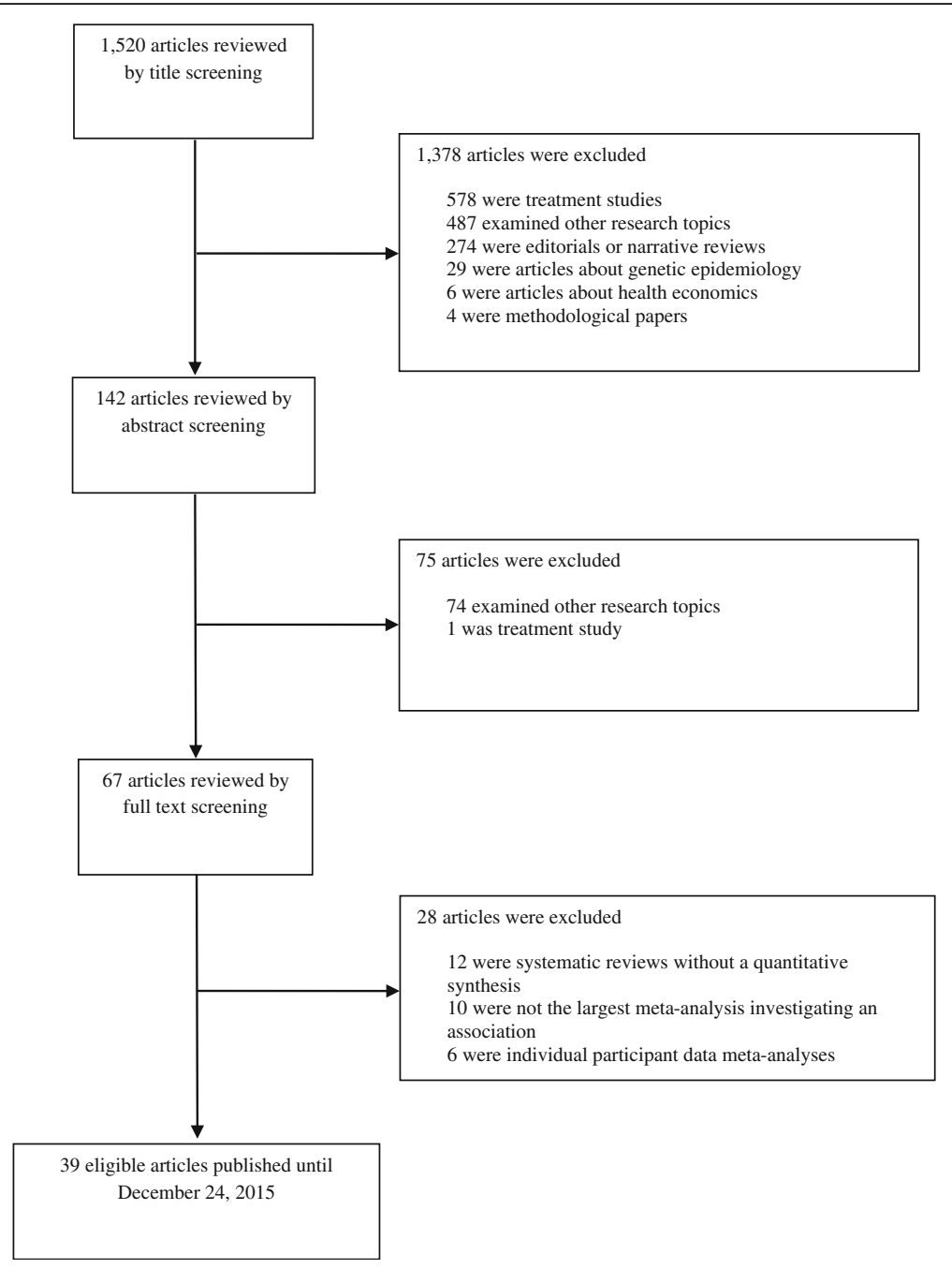

Fig. 1 Flow chart of literature search

Four meta-analyses (perinatal mortality in developing countries, coronary heart disease, school-age asthma, allcause mortality) had a $95 \%$ prediction interval excluding the null value. Five associations had large heterogeneity estimates $\left(\mathrm{I}^{2} \geq 50 \%\right.$ and $\left.\mathrm{I}^{2} \leq 75 \%\right)$, and 10 associations had very large heterogeneity estimates $\left(\mathrm{I}^{2}>75 \%\right)$. On bias assessment, seven associations had evidence for smallstudy effects (chronic kidney disease, coronary heart disease, diastolic blood pressure, intelligence in adolescence, medulloblastoma, wheezing disorders in childhood, and being overweight or obese in adulthood), and four associations (chronic kidney disease, diastolic blood pressure, intelligence in adolescence, and testicular cancer) had hints for excess significance bias (Table 1, Additional file 2: Table S2).

\section{Associations with high BW}

Across 18 meta-analyses examining high BW as a dichotomous trait, the median number of cases was 6115
(IQR, 3153-10,642), 16 meta-analyses were supported by more than 1000 cases, and the median number of datasets was 10 (IQR, 8-14). Ten associations presented a significant effect at $P<0.05$, but only three associations (acute lymphoblastic leukaemia, all types of leukaemia, and being overweight or obese in adulthood) remained statistically significant after the application of a more conservative significance threshold $\left(P<1 \times 10^{-6}\right)$. The largest study had a standard error of less than 0.10 in four meta-analyses and a more conservative effect compared to random-effects model in 12 meta-analyses. Only four meta-analyses (all types of leukaemia, neuroblastoma, type 1 diabetes mellitus, and being overweight or obese in adulthood) had a $95 \%$ prediction interval excluding the null value (Table 1 ). The heterogeneity estimate was large $\left(\mathrm{I}^{2} \geq 50 \%\right.$ and $\left.\mathrm{I}^{2} \leq 75 \%\right)$ in seven metaanalyses and only one meta-analysis presented very large heterogeneity $\left(\mathrm{I}^{2}>75 \%\right)$. Two associations presented hints for both small-study effects and excess significance 
Table 1 Quantitative synthesis, bias assessment and credibility assessment of 74 associations between different comparisons of birth weight and health outcomes or traits

\begin{tabular}{|c|c|c|c|c|c|c|c|c|c|c|c|}
\hline Reference & Outcome & $\begin{array}{l}\text { Level of } \\
\text { comparison }\end{array}$ & $\begin{array}{l}\mathrm{N} \text { cases/ } \\
\mathrm{N} \text { controls }\end{array}$ & $\begin{array}{l}\mathrm{N} \\
\text { datasets }\end{array}$ & $\begin{array}{l}\text { Effect size } \\
\text { metric }\end{array}$ & $\begin{array}{l}\text { Random-effects meta- } \\
\text { analysis ( } 95 \% \text { Cl) }\end{array}$ & $\begin{array}{l}P \text { value } \\
\text { (random) }\end{array}$ & $1^{2}$ & $\begin{array}{l}95 \% \\
\text { prediction } \\
\text { intervals }\end{array}$ & $\begin{array}{l}P \text { value for } \\
\text { Egger test }\end{array}$ & $\begin{array}{l}P \text { value for Excess } \\
\text { significance test }\end{array}$ \\
\hline $\begin{array}{l}\text { Araujo de } \\
\text { Franca, 2014 } \\
\text { [59] }\end{array}$ & Waist circumference & $\begin{array}{l}\text { Per } 1 \mathrm{~kg} \\
\text { increase }\end{array}$ & $4898^{d}$ & 6 & $\begin{array}{l}\text { Regression } \\
\text { coefficient }\end{array}$ & $-0.10(-0.73$ to 0.53$)$ & 0.760 & 73.2 & -2.04 to 1.84 & 0.725 & 0.943 \\
\hline $\begin{array}{l}\text { Araujo de } \\
\text { Franca, } 2014 \\
\text { [59] }\end{array}$ & Waist-to-hip ratio & $\begin{array}{l}\text { Per } 1 \mathrm{~kg} \\
\text { increase }\end{array}$ & $5008^{d}$ & 10 & $\begin{array}{l}\text { Regression } \\
\text { coefficient }\end{array}$ & $-0.59(-0.84$ to -0.34$)$ & $4.0 \times 10^{-6}$ & 0 & $\begin{array}{l}-0.89 \text { to } \\
-0.30\end{array}$ & 0.545 & 0.052 \\
\hline Baird, 2011 [48] & BMC in hip & $\begin{array}{l}\text { Per } 1 \mathrm{~kg} \\
\text { increase }\end{array}$ & $1795^{d}$ & 6 & $\begin{array}{l}\text { Regression } \\
\text { coefficient }\end{array}$ & $1.42(0.90$ to 1.94$)$ & $8.3 \times 10^{-8}$ & 5.9 & 0.56 to 2.28 & 0.584 & 0.838 \\
\hline Baird, 2011 [48] & BMC in lumbar spine & $\begin{array}{l}\text { Per } 1 \mathrm{~kg} \\
\text { increase }\end{array}$ & $3181^{d}$ & 7 & $\begin{array}{l}\text { Regression } \\
\text { coefficient }\end{array}$ & 1.72 (0.76 to 2.67 ) & $4.2 \times 10^{-4}$ & 33.5 & -0.53 to 3.97 & $0.069^{a}$ & $1.6 \times 10^{-4}$ \\
\hline Baird, 2011 [48] & BMD in hip & $\begin{array}{l}\text { Per } 1 \mathrm{~kg} \\
\text { increase }\end{array}$ & $3188^{d}$ & 7 & $\begin{array}{l}\text { Regression } \\
\text { coefficient }\end{array}$ & 0.01 (0.00 to 0.02 ) & 0.235 & 0 & -0.01 to 0.01 & 0.708 & 0.271 \\
\hline Baird, 2011 [48] & BMD in lumbar spine & $\begin{array}{l}\text { Per } 1 \mathrm{~kg} \\
\text { increase }\end{array}$ & $3506^{d}$ & 8 & $\begin{array}{l}\text { Regression } \\
\text { coefficient }\end{array}$ & $0.00(-0.01$ to 0.01$)$ & 0.779 & 14.9 & -0.02 to 0.02 & 0.831 & 0.341 \\
\hline $\begin{array}{l}\text { Berhan, } 2014 \\
{[46]}\end{array}$ & $\begin{array}{l}\text { Perinatal mortality in } \\
\text { developing countries }\end{array}$ & $\begin{array}{l}<2500 \mathrm{~g} \text { vs. } \\
\geq 2500 \mathrm{~g}\end{array}$ & $\begin{array}{l}21,184 / \\
285,970\end{array}$ & 14 & OR & 9.59 (6.11 to 15.04$)$ & $7.5 \times 10^{-23}$ & 98.9 & 1.43 to 64.18 & 0.954 & 0.707 \\
\hline $\begin{array}{l}\text { Caughey, } 2009 \\
\text { [13] }\end{array}$ & $\begin{array}{l}\text { Acute lymphoblastic } \\
\text { leukaemia }\end{array}$ & $\begin{array}{l}<2500 \mathrm{~g} \text { vs. } \\
\geq 2500 \mathrm{~g}\end{array}$ & $\begin{array}{l}4805 / \\
765,827\end{array}$ & 10 & $\mathrm{RR}$ & 0.97 (0.81 to 1.16$)$ & 0.736 & 0 & 0.78 to 1.20 & 0.289 & NP \\
\hline $\begin{array}{l}\text { Caughey, } 2009 \\
\text { [13] }\end{array}$ & $\begin{array}{l}\text { Acute lymphoblastic } \\
\text { leukaemia }\end{array}$ & $\begin{array}{l}>4000 \mathrm{~g} \text { vs. } \\
\leq 4000 \mathrm{~g}\end{array}$ & $\begin{array}{l}11,082 / \\
2,228,906\end{array}$ & 23 & $\mathrm{RR}$ & 1.29 (1.17 to 1.42$)$ & $1.9 \times 10^{-7}$ & 36.3 & 0.97 to 1.72 & $0.055^{\mathrm{a}}$ & $9.4 \times 10^{-7}$ \\
\hline $\begin{array}{l}\text { Caughey, } 2009 \\
\text { [13] }\end{array}$ & $\begin{array}{l}\text { Acute lymphoblastic } \\
\text { leukaemia }\end{array}$ & $\begin{array}{l}\text { Per } 1 \mathrm{~kg} \\
\text { increase }\end{array}$ & $\begin{array}{l}7404 / \\
858,650\end{array}$ & 16 & $\mathrm{RR}$ & 1.19 (1.10 to 1.28$)$ & $1.7 \times 10^{-5}$ & 54.7 & 0.93 to 1.52 & 0.990 & $2.2 \times 10^{-4}$ \\
\hline $\begin{array}{l}\text { Caughey, } 2009 \\
\text { [13] }\end{array}$ & All types of leukaemia & $\begin{array}{l}<2500 \mathrm{~g} \text { vs. } \\
\geq 2500 \mathrm{~g}\end{array}$ & $\begin{array}{l}5766 / \\
766,202\end{array}$ & 11 & RR & 1.04 (0.86 to 1.26$)$ & 0.683 & 10 & 0.76 to 1.43 & 0.357 & NP \\
\hline $\begin{array}{l}\text { Caughey, } 2009 \\
\text { [13] }\end{array}$ & All types of leukaemia & $\begin{array}{l}>4000 \mathrm{~g} \text { vs. } \\
\leq 4000 \mathrm{~g}\end{array}$ & NA/NA & 14 & $\mathrm{RR}$ & $1.42(1.26$ to 1.60$)$ & $1.2 \times 10^{-8}$ & 28.5 & 1.07 to 1.88 & $0.001^{\mathrm{a}}$ & NA \\
\hline $\begin{array}{l}\text { Caughey, } 2009 \\
\text { [13] }\end{array}$ & All types of leukaemia & $\begin{array}{l}\text { Per } 1 \mathrm{~kg} \\
\text { increase }\end{array}$ & $\begin{array}{l}11,313 / \\
861,710\end{array}$ & 21 & $\mathrm{RR}$ & 1.19 (1.12 to 1.27$)$ & $5.1 \times 10^{-8}$ & 45.3 & 0.97 to 1.45 & 0.590 & 0.001 \\
\hline $\begin{array}{l}\text { Caughey, } 2009 \\
\text { [13] }\end{array}$ & $\begin{array}{l}\text { Acute myeloid } \\
\text { leukaemia }\end{array}$ & $\begin{array}{l}<2500 \mathrm{~g} \text { vs. } \\
\geq 2500 \mathrm{~g}\end{array}$ & $\begin{array}{l}756 / \\
851,204\end{array}$ & 9 & RR & 1.46 (0.87 to 2.43 ) & 0.151 & 43.7 & 0.39 to 5.43 & 0.606 & NP \\
\hline $\begin{array}{l}\text { Caughey, } 2009 \\
\text { [13] }\end{array}$ & $\begin{array}{l}\text { Acute myeloid } \\
\text { leukaemia }\end{array}$ & $\begin{array}{l}>4000 \mathrm{~g} \text { vs. } \\
\leq 4000 \mathrm{~g}\end{array}$ & $\begin{array}{l}756 / \\
851,204\end{array}$ & 9 & $\mathrm{RR}$ & 1.25 (1.09 to 1.43$)$ & $1.3 \times 10^{-3}$ & 43.9 & 0.88 to 1.77 & 0.216 & $3.1 \times 10^{-4}$ \\
\hline Chen, 2012 [60] & Bone tumour & $\begin{array}{l}>4000 \mathrm{~g} \text { vs. } \\
\text { NBW }\end{array}$ & $\begin{array}{l}4044 / \\
272,354\end{array}$ & 8 & OR & 1.21 (0.97 to 1.50$)$ & 0.091 & 37.8 & 0.71 to 2.03 & $0.093^{\mathrm{a}}$ & 0.396 \\
\hline $\begin{array}{l}\text { Christian, } 2013 \\
{[40]}\end{array}$ & Childhood stunting & $\begin{array}{l}\text { SGA vs. AGA } \\
(B W \geq 2500 \mathrm{~g})\end{array}$ & $\begin{array}{l}5413 / \\
16,663\end{array}$ & 10 & OR & $1.92(1.75$ to 2.12$)$ & $1.1 \times 10^{-41}$ & 0 & 1.72 to 2.15 & 0.968 & NP \\
\hline $\begin{array}{l}\text { Christian, } 2013 \\
\text { [40] }\end{array}$ & Childhood stunting & $\begin{array}{l}\text { SGA vs. AGA } \\
(B W<2500 \mathrm{~g})\end{array}$ & $843 / 1171$ & 10 & OR & 3.00 (2.36 to 3.81$)$ & $2.3 \times 10^{-19}$ & 56.6 & 1.53 to 5.85 & 0.555 & NP \\
\hline
\end{tabular}


Table 1 Quantitative synthesis, bias assessment and credibility assessment of 74 associations between different comparisons of birth weight and health outcomes or traits (Continued)

\begin{tabular}{|c|c|c|c|c|c|c|c|c|c|c|c|}
\hline Cook, 2010 [58] & Testicular cancer & $\begin{array}{l}<2500 \mathrm{~g} \text { vs. } \\
\text { NBW }\end{array}$ & $\begin{array}{l}6906 / \\
612,741\end{array}$ & 17 & OR & 1.34 (1.08 to 1.67$)$ & $7.9 \times 10^{-3}$ & 50.9 & 0.69 to 2.62 & 0.135 & 0.049 \\
\hline $\begin{array}{l}\text { Davey-Smith, } \\
2007 \text { [61] }\end{array}$ & $\begin{array}{l}\text { Maternal cardiovascular } \\
\text { mortality }\end{array}$ & $\begin{array}{l}\text { Per } 1 \text { SD } \\
\text { increase }\end{array}$ & $\begin{array}{l}2976 / \\
974,667\end{array}$ & 6 & $\mathrm{HR}$ & 0.75 (0.67 to 0.84 ) & $3.1 \times 10^{-7}$ & 79.8 & 0.53 to 1.06 & 0.657 & 0.119 \\
\hline $\begin{array}{l}\text { Davey-Smith, } \\
2007 \text { [61] }\end{array}$ & $\begin{array}{l}\text { Paternal cardiovascular } \\
\text { mortality }\end{array}$ & $\begin{array}{l}\text { Per } 1 \text { SD } \\
\text { increase }\end{array}$ & $\begin{array}{l}9375 / \\
774,325\end{array}$ & 3 & $H R$ & 0.93 (0.91 to 0.95$)$ & $2.0 \times 10^{-9}$ & 0 & 0.80 to 1.09 & 0.317 & 0.516 \\
\hline $\begin{array}{l}\text { der Voort, } 2014 \\
\text { [8] }\end{array}$ & Pre-school wheezing & $\begin{array}{l}<2500 \mathrm{~g} \text { vs. } \\
\text { NBW }\end{array}$ & $\begin{array}{l}40,603 / \\
103,271\end{array}$ & 26 & OR & $1.10(1.00$ to 1.21$)$ & 0.051 & 16 & 0.89 to 1.36 & 0.829 & NP \\
\hline $\begin{array}{l}\text { der Voort, } 2014 \\
\text { [8] }\end{array}$ & School-age asthma & $\begin{array}{l}<2500 \mathrm{~g} \text { vs. } \\
\text { NBW }\end{array}$ & $\begin{array}{l}11,729 / \\
121,198\end{array}$ & 16 & OR & 1.13 (1.01 to 1.27$)$ & 0.032 & 0 & 1.00 to 1.28 & 0.639 & NP \\
\hline $\begin{array}{l}\text { Dodds, } 2012 \\
\text { [62] }\end{array}$ & Muscle strength & $\begin{array}{l}\text { Per } 1 \mathrm{~kg} \\
\text { increase }\end{array}$ & $20,461^{d}$ & 14 & $\begin{array}{l}\text { Regression } \\
\text { coefficient }\end{array}$ & 0.86 (0.58 to 1.15 ) & $1.9 \times 10^{-9}$ & 56.4 & -0.01 to 1.74 & 0.965 & NP \\
\hline $\begin{array}{l}\text { Harder, } 2007 \\
\text { [7] }\end{array}$ & $\begin{array}{l}\text { Type } 2 \text { diabetes } \\
\text { mellitus }\end{array}$ & $\begin{array}{l}<2500 \mathrm{~g} \text { vs. } \\
\geq 2500 \mathrm{~g}\end{array}$ & $\begin{array}{l}5815 / \\
100,759\end{array}$ & 10 & OR & 1.32 (1.06 to 1.64$)$ & 0.013 & 60.8 & 0.71 to 2.43 & 0.196 & NP \\
\hline $\begin{array}{l}\text { Harder, } 2007 \\
\text { [7] }\end{array}$ & $\begin{array}{l}\text { Type } 2 \text { diabetes } \\
\text { mellitus }\end{array}$ & $\begin{array}{l}>4000 \mathrm{~g} \text { vs. } \\
\leq 4000 \mathrm{~g}\end{array}$ & $\begin{array}{l}6005 / \\
108,400\end{array}$ & 9 & OR & 1.27 (1.01 to 1.59$)$ & 0.044 & 68.2 & 0.62 to 2.58 & 0.817 & NP \\
\hline $\begin{array}{l}\text { Harder, } 2008 \\
\text { [63] }\end{array}$ & Astrocytoma & $\begin{array}{l}<2500 \mathrm{~g} \text { vs. } \\
\geq 2500 \mathrm{~g}\end{array}$ & $\begin{array}{l}1574 / \\
160,146\end{array}$ & 6 & OR & 0.85 (0.58 to 1.25$)$ & 0.410 & 31.1 & 0.35 to 2.09 & 0.931 & NP \\
\hline $\begin{array}{l}\text { Harder, } 2008 \\
\text { [63] }\end{array}$ & Astrocytoma & $\begin{array}{l}>4000 \mathrm{~g} \text { vs. } \\
\leq 4000 \mathrm{~g}\end{array}$ & $\begin{array}{l}1812 / \\
1,649,625\end{array}$ & 8 & OR & 1.38 (1.07 to 1.79$)$ & 0.014 & 57.5 & 0.66 to 2.88 & 0.632 & 0.160 \\
\hline $\begin{array}{l}\text { Harder, } 2008 \\
\text { [63] }\end{array}$ & Medulloblastoma & $\begin{array}{l}<2500 \mathrm{~g} \text { vs. } \\
\geq 2500 \mathrm{~g}\end{array}$ & $\begin{array}{l}747 / \\
158,163\end{array}$ & 5 & OR & 1.65 (0.42 to 6.50$)$ & 0.475 & 88.2 & 0.01 to 223 & $0.828^{\mathrm{a}}$ & 0.503 \\
\hline $\begin{array}{l}\text { Harder, } 2008 \\
\text { [63] }\end{array}$ & Medulloblastoma & $\begin{array}{l}>4000 \mathrm{~g} \text { vs. } \\
\leq 4000 \mathrm{~g}\end{array}$ & $\begin{array}{l}853 / \\
1,647,552\end{array}$ & 7 & OR & 1.28 (1.02 to 1.59$)$ & 0.033 & 5.6 & 0.90 to 1.80 & 0.818 & NP \\
\hline $\begin{array}{l}\text { Harder, } 2009 \\
\text { [64] }\end{array}$ & $\begin{array}{l}\text { Type } 1 \text { diabetes } \\
\text { mellitus }\end{array}$ & $\begin{array}{l}<2500 \mathrm{~g} \text { vs. } \\
\geq 2500 \mathrm{~g}\end{array}$ & $\begin{array}{l}5236 / \\
1,385,809\end{array}$ & 8 & OR & 0.82 (0.55 to 1.24$)$ & 0.344 & 91.4 & 0.20 to 3.31 & 0.067 & NP \\
\hline $\begin{array}{l}\text { Harder, } 2009 \\
\text { [64] }\end{array}$ & $\begin{array}{l}\text { Type } 1 \text { diabetes } \\
\text { mellitus }\end{array}$ & $\begin{array}{l}>4000 \mathrm{~g} \text { vs. } \\
\leq 4000 \mathrm{~g}\end{array}$ & $\begin{array}{l}6406 / \\
2,388,046\end{array}$ & 10 & OR & 1.17 (1.09 to 1.26$)$ & $1.2 \times 10^{-5}$ & 0 & 1.08 to 1.28 & 0.883 & NP \\
\hline $\begin{array}{l}\text { Harder, } 2010 \\
\text { [65] }\end{array}$ & Neuroblastoma & $\begin{array}{l}<2500 \mathrm{~g} \text { vs. } \\
\geq 2500 \mathrm{~g}\end{array}$ & $\begin{array}{l}2907 / \\
2,156,535\end{array}$ & 10 & OR & 1.24 (0.99 to 1.55$)$ & 0.058 & 30.2 & 0.74 to 2.07 & 0.731 & NP \\
\hline $\begin{array}{l}\text { Harder, } 2010 \\
\text { [65] }\end{array}$ & Neuroblastoma & $\begin{array}{l}>4000 \mathrm{~g} \text { vs. } \\
\leq 4000 \mathrm{~g}\end{array}$ & $\begin{array}{l}2856 / \\
2,156,654\end{array}$ & 10 & OR & 1.19 (1.04 to 1.36$)$ & 0.013 & 0 & 1.01 to 1.39 & 0.517 & NP \\
\hline $\begin{array}{l}\text { Jackson } 2013 \\
\text { [66] }\end{array}$ & $\begin{array}{l}\text { Pneumonia in } \\
\text { childhood }\end{array}$ & $\begin{array}{l}<2500 \mathrm{~g} \text { vs. } \\
\geq 2500 \mathrm{~g}\end{array}$ & $1281 / 1464$ & 4 & OR & 3.18 (1.02 to 9.91$)$ & 0.046 & 95.6 & 0.01 to 714 & 0.366 & NP \\
\hline $\begin{array}{l}\text { Kormos, } 2013 \\
{[67]}\end{array}$ & $\begin{array}{l}\text { Intelligence in } \\
\text { adolescence }\end{array}$ & $\begin{array}{l}<2500 \mathrm{~g} \text { vs. } \\
\text { NBW }\end{array}$ & NA/NA & 15 & $O R^{c}$ & 0.35 (0.27 to 0.45$)$ & $1.7 \times 10^{-16}$ & 75.4 & 0.14 to 0.84 & $0.001^{\mathrm{a}}$ & $7.8 \times 10^{-4}$ \\
\hline $\begin{array}{l}\text { Lawlor } 2005 \\
{[68]}\end{array}$ & $\mathrm{FEV}_{1}$ & $\begin{array}{l}\text { Per } 1 \mathrm{~kg} \\
\text { increase }\end{array}$ & $5438^{d}$ & 7 & $\begin{array}{l}\text { Regression } \\
\text { coefficient }\end{array}$ & 0.06 (0.03 to 0.08$)$ & $1.4 \times 10^{-5}$ & 36.5 & -0.01 to 0.12 & 0.242 & $\mathrm{NP}$ \\
\hline $\begin{array}{l}\text { Lawlor } 2006 \\
\text { [69] }\end{array}$ & $\begin{array}{l}\text { Total cholesterol } \\
\text { in men }\end{array}$ & $\begin{array}{l}\text { Per } 1 \mathrm{~kg} \\
\text { increase }\end{array}$ & $33,650^{d}$ & 34 & $\begin{array}{l}\text { Regression } \\
\text { coefficient }\end{array}$ & $-0.04(-0.07$ to -0.01$)$ & 0.018 & 49.8 & -0.15 to 0.07 & 0.377 & NP \\
\hline
\end{tabular}


Table 1 Quantitative synthesis, bias assessment and credibility assessment of 74 associations between different comparisons of birth weight and health outcomes or traits (Continued)

\begin{tabular}{|c|c|c|c|c|c|c|c|c|c|c|c|}
\hline $\begin{array}{l}\text { Lawlor } 2006 \\
\text { [69] }\end{array}$ & Total cholesterol in women & $\begin{array}{l}\text { Per } 1 \mathrm{~kg} \\
\text { increase }\end{array}$ & $23,129^{d}$ & 34 & $\begin{array}{l}\text { Regression } \\
\text { coefficient }\end{array}$ & $-0.01(-0.04$ to 0.02$)$ & 0.510 & 27.7 & -0.09 to 0.07 & 0.686 & NP \\
\hline $\begin{array}{l}\text { Loret de Mola, } \\
2014 \text { [55] }\end{array}$ & Depression in adulthood & SGA vs. AGA & $397 / 2844$ & 5 & OR & 1.14 (0.64 to 2.03 ) & 0.656 & 49.3 & 0.20 to 6.36 & 0.791 & NP \\
\hline $\begin{array}{l}\text { Mebrahtu, } 2015 \\
\text { [11] }\end{array}$ & $\begin{array}{l}\text { Wheezing disorders in } \\
\text { childhood }\end{array}$ & $\begin{array}{l}<2500 \mathrm{~g} \text { vs. } \\
\geq 2500 \mathrm{~g}\end{array}$ & $\begin{array}{l}145,421 / \\
665,431\end{array}$ & 20 & OR & $1.61(1.39$ to 1.85$)$ & $1.1 \times 10^{-10}$ & 82.3 & 0.92 to 2.80 & $0.021^{\mathrm{a}}$ & NP \\
\hline $\begin{array}{l}\text { Mebrahtu, } 2015 \\
{[11]}\end{array}$ & $\begin{array}{l}\text { Wheezing disorders in } \\
\text { childhood }\end{array}$ & $\begin{array}{l}>4000 \mathrm{~g} \text { vs. } \\
\text { NBW }\end{array}$ & $\begin{array}{l}44,988 / \\
736,940\end{array}$ & 10 & OR & $1.02(1.00$ to 1.05$)$ & 0.100 & 0 & 0.99 to 1.05 & 0.985 & NP \\
\hline $\begin{array}{l}\text { Michos, } 2007 \\
\text { [70] }\end{array}$ & Testicular cancer & $\begin{array}{l}>4000 \mathrm{~g} \text { vs. } \\
\text { NBW }\end{array}$ & $\begin{array}{l}5684 / \\
123,120\end{array}$ & 10 & OR & 1.14 (0.99 to 1.31$)$ & 0.075 & 42.8 & 0.80 to 1.62 & 0.791 & 0.006 \\
\hline $\begin{array}{l}\text { Milne, } 2013 \\
\text { [56] }\end{array}$ & $\begin{array}{l}\text { Acute lymphoblastic } \\
\text { leukaemia }\end{array}$ & SGA vs. AGA & $\begin{array}{l}6835 / \\
11,689\end{array}$ & 12 & OR & $1.24(1.13$ to 1.36$)$ & $4.9 \times 10^{-6}$ & 0 & 1.12 to 1.37 & 0.101 & NP \\
\hline Mu, 2012 [71] & Diastolic blood pressure & $\begin{array}{l}<2500 \mathrm{~g} \text { vs. } \\
\geq 2500 \mathrm{~g}\end{array}$ & $29,192^{d}$ & 15 & $O R^{c}$ & 4.45 (1.32 to 14.99$)$ & 0.016 & 99.3 & 0.02 to 902 & $0.085^{\mathrm{a}}$ & 0.001 \\
\hline Mu, 2012 [71] & Systolic blood pressure & $\begin{array}{l}<2500 \mathrm{~g} \text { vs. } \\
\geq 2500 \mathrm{~g}\end{array}$ & $32,351^{d}$ & 19 & $O R^{c}$ & 7.45 (2.19 to 25.33$)$ & $1.3 \times 10^{-3}$ & 99.4 & 0.02 to 2296 & 0.148 & 0.615 \\
\hline $\mathrm{Mu}, 2014[41]^{\mathrm{b}}$ & Asthma in adulthood & $\begin{array}{l}<2500 \mathrm{~g} \text { vs. } \\
\geq 2500 \mathrm{~g}\end{array}$ & $\begin{array}{l}2111 / \\
37,409\end{array}$ & 4 & OR & $1.25(1.12$ to 1.40$)$ & $7.7 \times 10^{-5}$ & 0 & 0.98 to 1.60 & 0.884 & NP \\
\hline $\begin{array}{l}\text { Øglund, } 2015 \\
\text { [72] }\end{array}$ & Physical activity & $\begin{array}{l}\text { Per } 1 \mathrm{~kg} \\
\text { increase }\end{array}$ & $10,667^{d}$ & 8 & $\begin{array}{l}\text { Regression } \\
\text { coefficient }\end{array}$ & $-3.08(-10.20$ to 4.04$)$ & 0.397 & 9 & $\begin{array}{l}-14.81 \text { to } \\
8.66\end{array}$ & 0.881 & NP \\
\hline $\begin{array}{l}\text { Papadopoulou, } \\
2012 \text { [73] }\end{array}$ & $\begin{array}{l}\text { Hodgkin lymphoma in } \\
\text { childhood }\end{array}$ & $\begin{array}{l}<2500 \mathrm{~g} \text { vs. } \\
\text { NBW }\end{array}$ & $\begin{array}{l}669 / \\
64,058\end{array}$ & 3 & OR & 0.94 (0.54 to 1.65$)$ & 0.829 & 0 & 0.03 to 35.25 & 0.344 & NP \\
\hline $\begin{array}{l}\text { Papadopoulou, } \\
2012 \text { [73] }\end{array}$ & $\begin{array}{l}\text { Non-Hodgkin lymphoma in } \\
\text { childhood }\end{array}$ & $\begin{array}{l}<2500 \mathrm{~g} \text { vs. } \\
\text { NBW }\end{array}$ & $\begin{array}{l}1571 / \\
68,265\end{array}$ & 5 & OR & 1.07 (0.71 to 1.63$)$ & 0.740 & 55.4 & 0.29 to 3.95 & 0.599 & NP \\
\hline $\begin{array}{l}\text { Papadopoulou, } \\
2012[73]\end{array}$ & $\begin{array}{l}\text { Non-Hodgkin lymphoma in } \\
\text { childhood }\end{array}$ & $\begin{array}{l}>4000 \mathrm{~g} \text { vs. } \\
\text { NBW }\end{array}$ & $\begin{array}{l}1615 / \\
297,469\end{array}$ & 6 & OR & 1.17 (0.76 to 1.81$)$ & 0.473 & 66.7 & 0.32 to 4.28 & $0.085^{\mathrm{a}}$ & 0.207 \\
\hline $\begin{array}{l}\text { Panduru, } 2013 \\
\text { [74] }\end{array}$ & Atopic dermatitis & $\begin{array}{l}<2500 \mathrm{~g} \text { vs. } \\
\text { NBW }\end{array}$ & $\begin{array}{l}6315 / \\
100,663\end{array}$ & 10 & OR & 0.66 (0.48 to 0.90$)$ & $9.1 \times 10^{-3}$ & 85.6 & 0.23 to 1.87 & 0.558 & 0.412 \\
\hline $\begin{array}{l}\text { Panduru, } 2013 \\
\text { [74] }\end{array}$ & Atopic dermatitis & $\begin{array}{l}>4000 \mathrm{~g} \text { vs. } \\
\text { NBW }\end{array}$ & $\begin{array}{l}6224 / \\
62,672\end{array}$ & 6 & OR & 1.13 (0.97 to 1.31$)$ & 0.108 & 62.9 & 0.74 to 1.73 & 0.426 & NP \\
\hline $\begin{array}{l}\text { Risnes, } 2011 \\
{[42]^{\mathrm{b}}}\end{array}$ & All-cause mortality & $\begin{array}{l}<3000 \mathrm{~g} \text { vs. } \\
\text { NBW }\end{array}$ & $\begin{array}{l}32,926 / \\
276,648\end{array}$ & 8 & $\mathrm{HR}$ & $1.12(1.07$ to 1.16$)$ & $6.9 \times 10^{-8}$ & 21.7 & 1.03 to 1.21 & 0.642 & NP \\
\hline $\begin{array}{l}\text { Risnes, } 2011 \\
{[42]^{b}}\end{array}$ & All-cause mortality & $\begin{array}{l}>4000 \mathrm{~g} \text { vs. } \\
\text { NBW }\end{array}$ & $\begin{array}{l}32,926 / \\
276,648\end{array}$ & 8 & $H R$ & 1.02 (0.98 to 1.05$)$ & 0.302 & 0 & 0.98 to 1.06 & 0.317 & NP \\
\hline $\begin{array}{l}\text { Risnes, } 2011 \\
{[42]^{\mathrm{b}}}\end{array}$ & All-cause mortality & $\begin{array}{l}\text { Per } 1 \mathrm{~kg} \\
\text { increase }\end{array}$ & $\begin{array}{l}36,834 / \\
361,874\end{array}$ & 18 & $\mathrm{HR}$ & 0.94 (0.92 to 0.97 ) & $2.6 \times 10^{-6}$ & 18.6 & 0.89 to 0.99 & 0.838 & 0.038 \\
\hline $\begin{array}{l}\text { Risnes, } 2011 \\
{[42]^{\mathrm{b}}}\end{array}$ & $\begin{array}{l}\text { Mortality from cardiovascular } \\
\text { diseases }\end{array}$ & $\begin{array}{l}\text { Per } 1 \mathrm{~kg} \\
\text { increase }\end{array}$ & $\begin{array}{l}11,366 / \\
314,715\end{array}$ & 16 & $H R$ & 0.88 (0.85 to 0.91$)$ & $1.6 \times 10^{-13}$ & 0 & 0.84 to 0.91 & 0.717 & 0.177 \\
\hline $\begin{array}{l}\text { Risnes, } 2011 \\
{[42]^{b}}\end{array}$ & Mortality from cancer & $\begin{array}{l}\text { Per } 1 \mathrm{~kg} \\
\text { increase }\end{array}$ & $\begin{array}{l}9233 / \\
269,944\end{array}$ & 10 & $H R$ & $1.09(1.04$ to 1.14$)$ & $3.4 \times 10^{-4}$ & 20.8 & 0.99 to 1.19 & 0.276 & 0.037 \\
\hline
\end{tabular}


Table 1 Quantitative synthesis, bias assessment and credibility assessment of 74 associations between different comparisons of birth weight and health outcomes or traits (Continued)

\begin{tabular}{|c|c|c|c|c|c|c|c|c|c|c|c|}
\hline $\begin{array}{l}\text { Schellong, } 2012 \\
{[43]^{b}}\end{array}$ & $\begin{array}{l}\text { Overweight/obese in } \\
\text { adulthood }\end{array}$ & $\begin{array}{l}<2500 \mathrm{~g} \text { vs. } \\
\geq 2500 \mathrm{~g}\end{array}$ & $\begin{array}{l}73,420 / \\
330,648\end{array}$ & 30 & $\mathrm{OR}$ & 0.67 (0.59 to 0.76$)$ & $1.1 \times 10^{-9}$ & 82 & 0.38 to 1.18 & $0.079^{a}$ & NP \\
\hline $\begin{array}{l}\text { Schellong, } 2012 \\
{[43]^{b}}\end{array}$ & $\begin{array}{l}\text { Overweight/obese in } \\
\text { adulthood }\end{array}$ & $\begin{array}{l}>4000 \mathrm{~g} \text { vs. } \\
\leq 4000 \mathrm{~g}\end{array}$ & $\begin{array}{l}96,296 / \\
396,381\end{array}$ & 45 & OR & $1.68(1.58$ to 1.79$)$ & $3.6 \times 10^{-57}$ & 74.9 & 1.24 to 2.28 & 0.133 & NP \\
\hline $\begin{array}{l}\text { Silveira } 2008 \\
\text { [75] }\end{array}$ & Metabolic syndrome & $\begin{array}{l}<2500 \mathrm{~g} \text { vs. } \\
\text { NBW }\end{array}$ & NA/NA & 11 & OR & 2.54 (1.57 to 4.09$)$ & $1.4 \times 10^{-4}$ & 39.2 & 0.75 to 8.60 & 0.204 & NA \\
\hline Shi, 2015 [76] & $\begin{array}{l}\text { RSV-related acute lower } \\
\text { respiratory infection in } \\
\text { childhood }\end{array}$ & $\begin{array}{l}<2500 \mathrm{~g} \text { vs. } \\
\text { NBW }\end{array}$ & $\begin{array}{l}3383 / \\
155,872\end{array}$ & 5 & $\mathrm{OR}$ & 1.91 (1.45 to 2.53$)$ & $5.9 \times 10^{-6}$ & 59.1 & 0.81 to 4.54 & 0.881 & NP \\
\hline $\begin{array}{l}\text { van Montfoort, } \\
2005 \text { [77] }\end{array}$ & Cortisol levels & $\begin{array}{l}\text { Per } 1 \mathrm{~kg} \\
\text { increase }\end{array}$ & $2301^{d}$ & 11 & $\begin{array}{l}\text { Regression } \\
\text { coefficient }\end{array}$ & $-20.49(-35.97$ to -5.00$)$ & $9.5 \times 10^{-3}$ & 46.3 & $\begin{array}{l}-61.37 \text { to } \\
20.4\end{array}$ & 0.524 & NP \\
\hline Wang, 2014 [9] & Coronary heart disease & $\begin{array}{l}<2500 \mathrm{~g} \text { vs. } \\
\geq 2500 \mathrm{~g}\end{array}$ & $\begin{array}{l}13,089 / \\
360,209\end{array}$ & 16 & OR & $1.22(1.13$ to 1.31$)$ & $4.7 \times 10^{-7}$ & 7 & 1.08 to 1.37 & $0.039^{\mathrm{a}}$ & 0.751 \\
\hline Wang, 2014 [9] & Coronary heart disease & $\begin{array}{l}>4000 \mathrm{~g} \text { vs. } \\
\leq 4000 \mathrm{~g}\end{array}$ & $\begin{array}{l}18,243 / \\
313,235\end{array}$ & 14 & OR & 0.89 (0.81 to 0.98$)$ & 0.019 & 47.4 & 0.68 to 1.16 & 0.226 & NP \\
\hline Wang, 2014 [9] & Coronary heart disease & $\begin{array}{l}\text { Per } 1 \mathrm{~kg} \\
\text { increase }\end{array}$ & NA/NA & 23 & OR & 0.82 (0.78 to 0.86$)$ & $2.4 \times 10^{-15}$ & 41.1 & 0.70 to 0.96 & $0.014^{\mathrm{a}}$ & NA \\
\hline $\begin{array}{l}\text { Whincup, } 2008 \\
\text { [78] }\end{array}$ & Type 2 diabetes mellitus & $\begin{array}{l}\text { Per } 1 \mathrm{~kg} \\
\text { increase }\end{array}$ & $\begin{array}{l}6090 / \\
145,994\end{array}$ & 31 & OR & 0.80 (0.72 to 0.88$)$ & $1.8 \times 10^{-5}$ & 66.5 & 0.52 to 1.21 & 0.286 & 0.355 \\
\hline $\begin{array}{l}\text { White, } 2009 \\
\text { [79] }\end{array}$ & Chronic kidney disease & $\begin{array}{l}<2500 \mathrm{~g} \text { vs. } \\
\text { NBW }\end{array}$ & NA/NA & 21 & OR & 1.73 (1.44 to 2.08$)$ & $8.3 \times 10^{-9}$ & 66.3 & 0.88 to 3.38 & $0.015^{\mathrm{a}}$ & NA \\
\hline $\begin{array}{l}\text { Wojcik, } 2013 \\
{[14]^{\mathrm{b}}}\end{array}$ & Depression in adulthood & $\begin{array}{l}<2500 \mathrm{~g} \text { vs. } \\
\geq 2500 \mathrm{~g}\end{array}$ & $\begin{array}{l}9013 / \\
50,428\end{array}$ & 18 & OR & 1.15 (1.00 to 1.32$)$ & 0.057 & 34.3 & 0.79 to 1.67 & 0.171 & NP \\
\hline$X u, 2009[80]$ & Breast cancer & $\begin{array}{l}\text { Per } 1 \mathrm{~kg} \\
\text { increase }\end{array}$ & $\begin{array}{l}16,299 / \\
3,604,802\end{array}$ & 16 & OR & $1.08(1.03$ to 1.13$)$ & $2.8 \times 10^{-3}$ & 22.5 & 0.97 to 1.20 & 0.452 & $4.1 \times 10^{-4}$ \\
\hline Yang, 2014 [12] & Colorectal cancer & $\begin{array}{l}\text { Per } 1 \mathrm{~kg} \\
\text { increase }\end{array}$ & $\begin{array}{l}5985 / \\
723,087\end{array}$ & 5 & RR & 1.05 (0.93 to 1.19$)$ & 0.461 & 57.1 & 0.72 to 1.52 & 0.457 & 0.149 \\
\hline Yang, 2014 [12] & Endometrial cancer & $\begin{array}{l}\text { Per } 1 \mathrm{~kg} \\
\text { increase }\end{array}$ & $\begin{array}{l}3780 / \\
671,410\end{array}$ & 5 & RR & 0.91 (0.81 to 1.03$)$ & 0.128 & 40.2 & 0.65 to 1.27 & 0.867 & 0.758 \\
\hline Yang, 2014 [12] & Lung cancer & $\begin{array}{l}\text { Per } 1 \mathrm{~kg} \\
\text { increase }\end{array}$ & $\begin{array}{l}5207 / \\
696,742\end{array}$ & 4 & RR & 1.09 (1.02 to 1.16$)$ & 0.016 & 12.3 & 0.90 to 1.31 & 0.172 & 0.436 \\
\hline Yang, 2014 [12] & Melanoma & $\begin{array}{l}\text { Per } 1 \mathrm{~kg} \\
\text { increase }\end{array}$ & $\begin{array}{l}4000 / \\
3,821,122\end{array}$ & 6 & RR & $1.14(1.05$ to 1.24$)$ & $1.9 \times 10^{-3}$ & 0 & 1.01 to 1.29 & 0.912 & NP \\
\hline
\end{tabular}


Table 1 Quantitative synthesis, bias assessment and credibility assessment of 74 associations between different comparisons of birth weight and health outcomes or traits (Continued)

\begin{tabular}{|c|c|c|c|c|c|c|c|c|c|c|c|}
\hline Yang, 2014 [12] & Non-Hodgkin lymphoma & $\begin{array}{l}\text { Per } 1 \mathrm{~kg} \\
\text { increase }\end{array}$ & $\begin{array}{l}2056 / \\
626,082\end{array}$ & 3 & $\mathrm{RR}$ & $1.12(1.01$ to 1.24$)$ & 0.033 & 0 & 0.58 to 2.15 & 0.487 & NP \\
\hline Yang, 2014 [12] & Ovarian cancer & $\begin{array}{l}\text { Per } 1 \mathrm{~kg} \\
\text { increase }\end{array}$ & $\begin{array}{l}2880 / \\
805,887\end{array}$ & 5 & RR & 0.96 (0.88 to 1.04$)$ & 0.295 & 0 & 0.83 to 1.10 & 0.609 & NP \\
\hline $\begin{array}{l}\text { Zhang, } 2013 \\
\text { [81] }\end{array}$ & Diastolic blood pressure & $\begin{array}{l}>4000 \mathrm{~g} \text { vs. } \\
\text { NBW }\end{array}$ & $150,980^{d}$ & 23 & $\mathrm{MD}$ & 0.19 ( -0.23 to 0.62$)$ & 0.367 & 72.5 & -1.39 to 1.79 & 0.792 & NP \\
\hline $\begin{array}{l}\text { Zhang, } 2013 \\
\text { [81] }\end{array}$ & Systolic blood pressure & $\begin{array}{l}>4000 \mathrm{~g} \text { vs. } \\
\text { NBW }\end{array}$ & $151,935^{d}$ & 24 & $\mathrm{MD}$ & $-0.25(-0.92$ to 0.42$)$ & 0.466 & 79.3 & -2.97 to 2.47 & 0.477 & NP \\
\hline
\end{tabular}

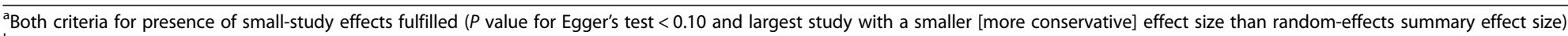

${ }^{\mathrm{b}}$ The highlighted papers performed a comparison between studies adjusting for and not adjusting for gestational age

'Random-effects summary effect size estimated from standardized mean difference transformed to odds ratio

IIn meta-analyses with a continuous outcome, the total sample size is reported

$A G A$ adequate-for-gestational age, $B M C$ bone mineral concentration, $B M D$ bone mineral density, $C l$ confidence interval, $F E V$, forced expiratory volume in the first second, $H R$ hazard ratio, $O R$ odds ratio, $R R$ risk ratio, $M D$ mean difference, $N A$ not available, $N B W$ normal birth weight, $N P$ not pertinent (because the number of expected significant studies was larger than the number of observed significant studies), $R S V$ respiratory syncytial virus, SD standard error, SGA small-for-gestational age 
bias (acute lymphoblastic leukaemia and all types of leukaemia), another two associations had only smallstudy effects (bone tumour and non-Hodgkin lymphoma in childhood), and two additional associations had hints for excess significance bias (acute myeloid leukaemia and testicular cancer).

\section{Dose-response associations with BW}

Across 28 meta-analyses, the median number of cases was 6747 (IQR, 3945-11,326) and the median number of datasets was 8 (IQR, 6-16). Overall, 17 associations were significant at $P<0.05$, but only six associations survived in the application of a more stringent $P$ value $\left(P<1 \times 10^{-6}\right)$. The largest study had a standard error of less than 0.10 in 21 meta-analyses and a more conservative effect compared to the random-effects model in 20 meta-analyses. Only six associations (all-cause mortality, bone mineral concentration in hip, coronary heart disease, melanoma, mortality from cardiovascular diseases, and waist-to-hip ratio) presented $95 \%$ prediction interval excluding the null value (Table 1). Five associations presented large heterogeneity, and one association had very large heterogeneity. Hints for small-study effects and excess statistical significance were present in two (bone mineral concentration in lumbar spine, coronary heart disease) and eight meta-analyses (all-cause mortality, acute lymphoblastic leukaemia, all types of leukaemia, bone mineral concentration in lumbar spine, breast cancer, coronary heart disease, mortality from cancer, and waist-to-hip ratio), respectively (Table 1 , Additional file 2 : Table S2).

\section{BW relative to gestational age}

Three papers performed four meta-analyses examining associations between small-for-gestational-age infants (defined as BW below the 10th percentile for the gestational age) and the risk for acute lymphoblastic leukaemia, childhood stunting and depression. No meta-analyses on largefor-gestational age infants were identified. Under the random-effects model, three associations had a statistically significant effect at $P<1 \times 10^{-6}$ and $95 \%$ prediction interval excluding the null value (acute lymphoblastic leukaemia and childhood stunting in infants with low and normal BW; Table 1). Only one association had large between-study heterogeneity, whereas none of the examined associations presented evidence for small-study effects or excess significance bias.

Despite the importance of gestational age on BW, only four out of the 36 papers (pertained to seven metaanalyses) examining low BW, high BW or dose-response relationships with $\mathrm{BW}$, presented subgroup analyses, including only studies that provided gestational ageadjusted estimates (Table 1) [14, 41-43]. None of these analyses observed a statistically significant difference in the summary effect between the studies adjusting for gestational age and the unadjusted studies. Additionally, 18 (46\%) papers mentioned that the observed effect might differ from the true effect because gestational age was not considered as an adjustment variable in several observational studies. Twenty papers (51 \%) reported the observational studies that adjusted for gestational age in the statistical models.

\section{Assessment of epidemiological credibility}

Twenty-eight of 78 associations (36\%) did not present a significant summary effect at $P<0.05$. Of the remaining 50 associations, only four presented convincing evidence by having more than 1000 cases, not large heterogeneity, $95 \%$ prediction interval excluding the null value, a significant summary effect at $P<1 \times 10^{-6}$, and absence of small-study effects and excess significance bias (Table 2). These associations pertained to all-cause mortality for low versus normal $\mathrm{BW}$, bone mineral concentration in hip and mortality from cardiovascular diseases per $1 \mathrm{~kg}$ increase in BW, and childhood stunting for small- versus adequate-for-gestational-age infants with $B W \geq 2500$ g. Notably, apart from the meta-analyses on stunting, which included gestational age in the definition of the examined phenotype (small-for-gestational-age), none of the other three meta-analyses with convincing evidence restricted their analyses to studies with adjustment for gestational age. Eleven additional associations had highly suggestive evidence (more than 1000 cases, a significant summary effect at $P<1 \times 10^{-6}$ and largest study with a significant effect). These associations examined perinatal mortality in developing countries, wheezing disorders, being overweight or obese in adulthood, coronary heart disease for the comparison of $<2500 \mathrm{~g}$ versus $\geq 2500 \mathrm{~g}$, intelligence in adolescence for the comparison of low BW versus normal BW, all types of leukaemia, being overweight or obese in adulthood for the comparison of $>4000 \mathrm{~g}$ versus $\leq 4000 \mathrm{~g}$, muscle strength and coronary heart disease for the comparison of increase per $1 \mathrm{~kg}$ in BW, and maternal cardiovascular mortality and paternal cardiovascular mortality for the comparison of increase per $1 \mathrm{SD}$ in BW. Fourteen associations presented suggestive evidence and 13 associations had weak evidence (Table 2).

\section{Discussion}

Our work constitutes the first comprehensive mapping and appraisal of the association between BW and the risk of subsequent health outcomes, as provided by published systematic reviews and meta-analyses of observational studies. Overall, 78 associations have been examined, including a diverse range of outcomes: cardiovascular, cancer, metabolic, respiratory and mortality outcomes, and disease traits and biomarkers. Despite 
Table 2 Summary of evidence grading for meta-analyses associating different contrasts of birth weight and risk of future disease

\begin{tabular}{|c|c|c|c|c|c|c|c|c|}
\hline \multirow[t]{2}{*}{$\begin{array}{l}\text { Level of } \\
\text { evidence }\end{array}$} & \multirow[t]{2}{*}{ Criteria } & \multicolumn{2}{|c|}{$\begin{array}{l}\text { Low birth weight vs. normal } \\
\text { birth weight }\end{array}$} & \multicolumn{2}{|c|}{$\begin{array}{l}\text { High birth weight vs. normal } \\
\text { birth weight }\end{array}$} & \multicolumn{2}{|c|}{ Per unit/SD increase } & \multirow{2}{*}{$\begin{array}{l}\text { SGA vs. AGA } \\
\text { Increased risk in SGA } \\
\text { group }\end{array}$} \\
\hline & & $\begin{array}{l}\text { Increased risk in } \\
\text { LBW group }\end{array}$ & $\begin{array}{l}\text { Decreased } \\
\text { risk in LBW } \\
\text { group }\end{array}$ & $\begin{array}{l}\text { Increased risk in } \\
\text { HBW group }\end{array}$ & $\begin{array}{l}\text { Decreased } \\
\text { risk in } \\
\text { HBW } \\
\text { group }\end{array}$ & $\begin{array}{l}\text { Increased risk } \\
\text { per unit/SD } \\
\text { increase }\end{array}$ & $\begin{array}{l}\text { Decreased risk } \\
\text { per unit/SD } \\
\text { increase }\end{array}$ & \\
\hline Convincing & $\begin{array}{l}>1000 \text { cases, } \\
P<1 \times 10^{-6}, \\
1^{2}<50 \%, \\
95 \% \mathrm{PI} \\
\text { excluding } \\
\text { the null } \\
\text { value, no } \\
\text { small-study } \\
\text { effects and } \\
\text { excess sig- } \\
\text { nificance } \\
\text { bias }\end{array}$ & $\begin{array}{l}\text { All-cause } \\
\text { mortality }\end{array}$ & None & None & None & BMC in hip & $\begin{array}{l}\text { Mortality from } \\
\text { cardiovascular } \\
\text { diseases }\end{array}$ & $\begin{array}{l}\text { Childhood stunting } \\
\text { (in BW } \geq 2500 \text { g group) }\end{array}$ \\
\hline $\begin{array}{l}\text { Highly } \\
\text { suggestive }\end{array}$ & $\begin{array}{l}>1000 \text { cases, } \\
P<1 \times 10^{-6}, \\
\text { largest study } \\
\text { with a } \\
\text { statistically } \\
\text { significant } \\
\text { effect }\end{array}$ & $\begin{array}{l}\text { Perinatal } \\
\text { mortality in } \\
\text { developing } \\
\text { countries, } \\
\text { wheezing } \\
\text { disorders in } \\
\text { childhood, } \\
\text { coronary heart } \\
\text { disease }\end{array}$ & $\begin{array}{l}\text { Overweight } \\
\text { or obese in } \\
\text { adulthood, } \\
\text { intelligence } \\
\text { in } \\
\text { adolescence }\end{array}$ & $\begin{array}{l}\text { All types of } \\
\text { leukaemia, } \\
\text { overweight or } \\
\text { obese in } \\
\text { adulthood }\end{array}$ & None & None & $\begin{array}{l}\text { Muscle } \\
\text { strength, } \\
\text { coronary heart } \\
\text { disease, } \\
\text { maternal } \\
\text { cardiovascular } \\
\text { mortality, } \\
\text { paternal } \\
\text { cardiovascular } \\
\text { mortality }\end{array}$ & None \\
\hline Suggestive & $\begin{array}{l}>1000 \text { cases } \\
P<1 \times 10^{-3}\end{array}$ & $\begin{array}{l}\text { Asthma in } \\
\text { adulthood, RSV- } \\
\text { related acute } \\
\text { lower respira- } \\
\text { tory infections } \\
\text { in childhood, } \\
\text { chronic kidney } \\
\text { disease }\end{array}$ & None & $\begin{array}{l}\text { Acute } \\
\text { lymphoblastic } \\
\text { leukaemia, type } 1 \\
\text { diabetes mellitus }\end{array}$ & None & $\begin{array}{l}\text { BMC in } \\
\text { lumbar spine, } \\
\text { acute } \\
\text { lymphoblastic } \\
\text { leukaemia, all } \\
\text { types of } \\
\text { leukaemia, } \\
\text { FEV } \text {, mortality }_{\text {from cancer }}\end{array}$ & $\begin{array}{l}\text { Waist-to-hip } \\
\text { ratio, all-cause } \\
\text { mortality, type } \\
2 \text { diabetes } \\
\text { mellitus }\end{array}$ & $\begin{array}{l}\text { Acute lymphoblastic } \\
\text { leukaemia }\end{array}$ \\
\hline Weak & $\begin{array}{l}\text { The } \\
\text { remaining } \\
\text { associations } \\
\text { with } P<0.05\end{array}$ & $\begin{array}{l}\text { Diastolic blood } \\
\text { pressure, } \\
\text { testicular } \\
\text { cancer, type } 2 \\
\text { diabetes } \\
\text { mellitus, } \\
\text { metabolic } \\
\text { syndrome, } \\
\text { pneumonia in } \\
\text { childhood, } \\
\text { school-age } \\
\text { asthma, systolic } \\
\text { blood pressure }\end{array}$ & $\begin{array}{l}\text { Atopic } \\
\text { dermatitis }\end{array}$ & $\begin{array}{l}\text { Acute myeloid } \\
\text { leukaemia, } \\
\text { astrocytoma, } \\
\text { medulloblastoma, } \\
\text { neuroblastoma, } \\
\text { type } 2 \text { diabetes } \\
\text { mellitus }\end{array}$ & $\begin{array}{l}\text { Coronary } \\
\text { heart } \\
\text { disease }\end{array}$ & $\begin{array}{l}\text { Breast cancer } \\
\text { at any age, } \\
\text { lung cancer, } \\
\text { melanoma, } \\
\text { non-Hodgkin } \\
\text { lymphoma }\end{array}$ & $\begin{array}{l}\text { Cortisol levels, } \\
\text { total } \\
\text { cholesterol in } \\
\text { men }\end{array}$ & $\begin{array}{l}\text { Childhood stunting } \\
\text { (in BW }<2500 \text { g group) }\end{array}$ \\
\hline
\end{tabular}

$A G A$ adequate-for-gestational age, $B M C$ bone mineral concentration, $B W$ birth weight, $F E V_{1}$ forced expiratory volume in the first second, $H B W$ high birth weight, $L B W$ low birth weight, $S D$ standard deviation, $S G A$ small-for-gestational age, $R S V$ respiratory syncytial virus

common belief that the intrauterine environment as assessed by BW is associated with many diseases and disease traits in adult life [1, 6-10], our comprehensive assessment shows that convincing evidence only exists between the associations of low BW and increased risk for all-cause mortality, per $1 \mathrm{~kg}$ increase in $\mathrm{BW}$ and higher bone mineral concentration in hip and lower risk for mortality from cardiovascular diseases. Furthermore, the association between small-for-gestational-age and childhood stunting in low- and middle-income countries was supported by convincing evidence. There was no convincing evidence supporting associations between high BW and later outcomes; however, the associations with overweight or obesity in later life and all types of leukaemia were highly suggestive.

The associations between BW and cardiovascular disease were amongst the first to be observed in the medical literature [1-5] and our data suggests that the 
current evidence is highly suggestive. Both metaanalyses looking at low $(<2500 \mathrm{~g})$ versus high $(\geq 4000 \mathrm{~g})$ $\mathrm{BW}$ and those examining per $1 \mathrm{SD}$ increase in $\mathrm{BW}$ showed highly significant summary effects and small between-study heterogeneity. However, both associations presented evidence for small-study effects and the doseresponse association additionally had hints for excess significance bias. The latter may have resulted in inflated effect estimates for an association with cardiovascular disease that needs cautious interpretation [35, 44]. Despite the fact that studies have adjusted for a range of confounders, including socioeconomic status, not all studies were adjusted for gestational age, which is an important confounder and this, as well as other unrecognized confounders, could explain the observed association. In addition, the mechanisms underlying this association remain unclear despite many hypotheses having been suggested, including the one supporting that intrauterine under-nutrition leads to fetal adaptation, which is subsequently related to adverse cardiovascular risk in later life [10]. However, others have provided evidence that at least some of the association between the BW of individuals and their later risk of cardiovascular disease may be genetic and therefore not modifiable via interventions that target the intrauterine environment [45]. The causal pathway linking BW to cardiovascular risk needs further elucidation to allow evidence-based public health interventions.

The observed increased risk of cardiovascular disease associated with lower BW is likely to be a main contributor to the inverse association of BW with all-cause mortality; an association supported by convincing evidence in our assessment [42]. The higher incidence of perinatal mortality in the low BW group is also likely contributing to the all-cause mortality association with low BW, but only to a small extent. Babies born with a BW below $2500 \mathrm{~g}$ had increased perinatal mortality, an association supported by a very large summary effect estimate and a very small $P$ value [46]. However, the metaanalysis on perinatal mortality was focused exclusively on developing countries. Therefore, the effect estimate might be exaggerated due to lack of neonatal intensive care units or difficult access to specialized healthcare facilities in these countries [47]. These data could not be generalised to other settings where high-quality healthcare is available.

The association between low BW and low bone mineral concentration in later life is less well studied compared to other outcomes and current data stem from six studies contributing to the meta-analysis [48]. Despite the fact that the association with bone mineral concentration in hip showed convincing evidence, cautious interpretation is required as data on osteoporotic fractures has not been reviewed and meta-analyses on other anatomical sites (e.g. lumbar spine) showed evidence for excess significance bias and no convincing associations.

Comparisons between BW and later overweight and obesity do not support a detrimental health effect of low $\mathrm{BW}$. BW less than $2500 \mathrm{~g}$ was found to be protective for being overweight or obese, whereas BW greater than $4000 \mathrm{~g}$ was linked with an increased risk for being overweight or obese in adult life [43]. These associations were supported by highly suggestive evidence, but they also displayed very large between-study heterogeneity. Heterogeneity could be due to biased results in some of the included studies, but it could also reflect genuine differences across studies [35]. BW distributions are remarkably different across developed and developing countries [49], and the associations between BW and later adiposity may differ in these populations, contributing to the heterogeneity of the observed results. High $\mathrm{BW}$ is potentially causally associated with maternal BMI and glucose levels $[50,51]$; however, the extent to which it could be modified through lifestyle or pharmacological interventions merits further investigation, particularly through long-term follow-up of interventions during pregnancy, which will strengthen and enhance the available evidence, particularly between high BW and subsequent risk of childhood and adulthood obesity [52-54].

Although 29 associations focused on outcomes related to different types of cancer, high BW was found to be a risk factor only for developing leukaemia [13]. The associated summary effect estimate might be inflated by the presence of small-study effects and excess significance bias. However, the statistical heterogeneity was not large, the $95 \%$ prediction interval excluded the null value and the association was highly significant. Similarly, despite diabetes being central in the "fetal origin hypothesis" [7], its association with high and low BW has weak evidence in the literature and is only suggestive of a direct association with high BW in line with the obesity-associated evidence.

Despite intensive research on BW reflected by the large number of meta-analyses identified, there were only three papers that performed meta-analyses of studies assessing low BW in relation to gestational age $[40,55,56]$, whereas no single meta-analysis on large-for-gestational-age neonates was identified. As BW and gestational age are highly correlated, analyses which consider size-forgestational-age rather than BW adjusted for gestational age have been proposed as a more appropriate alternative [57, 58]. Among the examined phenotypes in relation to small-for-gestational-age, the association between small-for-gestational-age without low BW and childhood stunting in low- and middle-income countries showed convincing evidence. However, those results require cautious interpretation as the analyses were stratified by BW and the association between 
small-for-gestational age with low BW and childhood stunting showed a much weaker effect estimate and was only supported by weak evidence. Additionally, those analyses focused on low- and middle-income countries, limiting the generalisability of those results but at the same time also highlighting the need for interventions during the pregnancy period in these populations [40]. The remaining meta-analyses included a mixture of studies that adjusted or not their analyses for gestational age and, hence, the current literature is inconclusive on the effects of BW relative to gestational age.

In the present study, we applied the umbrella review approach summarising data from already published systematic reviews and meta-analyses. This approach takes full advantage of the existing meta-analyses to perform a standardised methodological process for the assessment of the epidemiological credibility of the findings. However, our study has some caveats. First, the Egger test and excess statistical significance test offer hints of bias, and not proof thereof, while the Egger test is difficult to interpret when the between-study heterogeneity is large. Further, our excess significance estimates were based on the largest study of each meta-analysis and they might be conservative, because often these studies were not necessarily very large or might have had inherent biases themselves. Furthermore, we did not appraise the quality of the primary studies, because this was beyond the scope of this umbrella review. This should be the aim of the original systematic reviews and meta-analyses, which should examine the methodological characteristics of the component studies.

\section{Conclusions}

Our study maps the current status of evidence on 78 associations of BW with various health outcomes, traits and biomarkers. Of them, only three examined the effects of BW in relation to gestational age through size-at-birth defined phenotypes. Our results show that the range of outcomes associated with BW is narrow and smaller than described under the fetal origin of disease hypothesis. Currently, there is weak evidence that BW constitutes an effective public policy intervention for long-term health and disease.

\section{Additional files}

Additional file 1: Table S1. Overlapping associations examined in older papers and/or under different levels of comparison. BMC: bone mineral concentration, BMD: bone mineral density, BMI: body mass index, MD: mean difference, NBW: normal birth weight, OR: odds ratio, RR: risk ratio [82-90]. (DOC $229 \mathrm{~kb})$

Additional file 2: Table S2. Comparison of random-effects summary effect size and largest study effect size, expected and observed number of significant associations and excess significance test in each meta-analysis. tRandom-effects summary effect size estimated from standardized mean difference transformed to odds ratio. AGA: adequate-for-gestational age, BMC: bone mineral concentration, BMD: bone mineral density, Cl: confidence interval, FEV: forced expiratory volume in the first second, HR: hazard ratio, OR: odds ratio, RR: risk ratio, MD: mean difference, RSV: respiratory syncytial virus, SD: standard error, SE: standard error, SGA: small-forgestational age. (DOC $447 \mathrm{~kb}$ )

Abbreviations

BW: Birth weight; IQR: Inter-quartile range

\section{Acknowledgements}

Not applicable.

\section{Funding}

The authors declare that they did not receive any financial support for the present study.

\section{Availability of data and materials}

The datasets analysed and generated during the current study are available from the corresponding author on reasonable request.

\section{Authors' contributions}

IT and EE designed the study. LB and CK performed the literature search, the selection of eligible articles and the data extraction. LB analyzed the data. LB, MDS, EE and IT were involved in data interpretation. LB and IT wrote the first draft of the manuscript and LB, MDS, EE and IT were involved in the revision of the manuscript. All authors approved the final version of the submitted manuscript.

\section{Competing interests}

The authors declare that they have no competing interests.

\section{Consent for publication}

Not applicable.

Ethics approval and consent to participate

Not applicable.

\section{Author details}

${ }^{1}$ Department of Hygiene and Epidemiology, University of Ioannina Medical School, loannina, Greece. ${ }^{2}$ Academic Department of Obstetrics and Gynecology, Chelsea and Westminster Hospital, Imperial College London, London, UK. ${ }^{3}$ Department of Epidemiology and Biostatistics, School of Public Health, Imperial College London, London, UK. ${ }^{4}$ MRC-PHE Centre for Environment and Health, School of Public Health, Imperial College London, London, UK.

Received: 16 June 2016 Accepted: 9 September 2016

Published online: 28 September 2016

\section{References}

1. Hales C, Barker D. The thrifty phenotype hypothesis. Br Med Bull. 2001;60:5-20.

2. Barker D, Osmond C, Golding J, Kuh D, Wadsworth M. Growth in utero, blood pressure in childhood and adult life, and mortality from cardiovascular disease. BMJ. 1989;298:564-7.

3. Barker DJ, Winter PD, Osmond C, Margetts B, Simmonds SJ. Weight in infancy and death from ischaemic heart disease. Lancet. 1989;2:577-80.

4. Stein CE, Fall CH, Kumaran K, Osmond C, Cox V, Barker DJ. Fetal growth and coronary heart disease in south India. Lancet. 1996;348:1269-73.

5. Barker DJ, Osmond C. Infant mortality, childhood nutrition, and ischaemic heart disease in England and Wales. Lancet. 1986;1(8489):1077-81.

6. Barker DJ. Fetal origins of coronary heart disease. BMJ. 1995;311:171-4.

7. Harder T, Rodekamp E, Schellong K, Dudenhausen JW, Plagemann A. Birth weight and subsequent risk of type 2 diabetes: a meta-analysis. Am J Epidemiol. 2007;165:849-57.

8. Der Voort AMM S-V, Arends LR, De Jongste JC, Annesi-Maesano I, Arshad $\mathrm{SH}$, Barros $\mathrm{H}$, et al. Preterm birth, infant weight gain, and childhood asthma risk: a meta-analysis of 147,000 European children. J Allergy Clin Immunol. 2014;133:1317-29.

9. Wang S-F, Shu L, Sheng J, Mu M, Wang S, Tao X-Y, et al. Birth weight and risk of coronary heart disease in adults: a meta-analysis of prospective cohort studies. J Dev Orig Health Dis. 2014;5:408-19. 
10. Godfrey KM, Barker DJ. Fetal programming and adult health. Public Health Nutr. 2001;:611-24.

11. Mebrahtu TF, Feltbower RG, Greenwood DC, Parslow RC. Birth weight and childhood wheezing disorders: a systematic review and meta-analysis. J Epidemiol Community Heal. 2015;69:500-8.

12. Yang TO, Reeves GGK, Green J, Beral V, Cairns BBJ, Abbiss H, et al. Birth weight and adult cancer incidence: large prospective study and metaanalysis. Ann Oncol. 2014;25:1836-43.

13. Caughey RW, Michels KB. Birth weight and childhood leukemia: a metaanalysis and review of the current evidence. Int J Cancer. 2009;124:2658-70.

14. Wojcik W, Lee W, Colman I, Hardy R, Hotopf M. Foetal origins of depression? A systematic review and meta-analysis of low birth weight and later depression. Psychol Med. 2013;43:1-12.

15. World Health Organization. Global Nutrition Targets 2025: Low birth weight policy brief. Geneva: WHO; 2014.

16. Wilcox A. On the importance-and the unimportance-of birthweight. Int J Epidemiol. 2001;30:1233-41.

17. Ioannidis JPA. Integration of evidence from multiple meta-analyses: a primer on umbrella reviews, treatment networks and multiple treatments metaanalyses. CMAJ. 2009;181:488-93.

18. Theodoratou E, Tzoulaki I, Zgaga L, loannidis JPA. Vitamin D and multiple health outcomes: umbrella review of systematic reviews and meta-analyses of observational studies and randomised trials. BMJ. 2014;348:92035.

19. Belbasis L, Bellou V, Evangelou E, loannidis JPA, Tzoulaki I. Environmental risk factors and multiple sclerosis: an umbrella review of systematic reviews and meta-analyses. Lancet Neurol. 2015;14:263-73.

20. Bellou V, Belbasis L, Tzoulaki I, Evangelou E, loannidis JPA. Environmental risk factors and Parkinson's disease: An umbrella review of meta-analyses. Parkinsonism Relat Disord. 2016;23:1-9.

21. Belbasis L, Bellou V, Evangelou E. Environmental risk factors and amyotrophic lateral sclerosis: an umbrella review and critical assessment of current evidence from systematic reviews and meta-analyses of observational studies. Neuroepidemiology. 2016;46:96-105.

22. Gale CR, Sayer AA, Cooper C, Dennison EM, Starr JM, Whalley LJ, et al. Factors associated with symptoms of anxiety and depression in five cohorts of community-based older people: the HALCyon (Healthy Ageing across the Life Course) Programme. Psychol Med. 2011;41:2057-73.

23. Cardwell CR, Stene LC, Joner G, Davis EA, Cinek O, Rosenbauer J, et al. Birthweight and the risk of childhood-onset type 1 diabetes: a meta-analysis of observational studies using individual patient data. Diabetologia. 2010;53:641-51.

24. Andersen LG, Angquist L, Gamborg M, Byberg L, Bengtsson C, Canoy D, et al. Birth weight in relation to leisure time physical activity in adolescence and adulthood: meta-analysis of results from 13 Nordic cohorts. PLoS One. 2009;4:e8192.

25. Gamborg M, Byberg L, Rasmussen F, Andersen PK, Baker JL, Bengtsson C, et al. Birth weight and systolic blood pressure in adolescence and adulthood: meta-regression analysis of sex- and age-specific results from 20 Nordic studies. Am J Epidemiol. 2007:166:634-45.

26. Mirabello L, Pfeiffer R, Murphy G, Daw NC, Patiño-Garcia A, Troisi RJ, et al. Height at diagnosis and birth-weight as risk factors for osteosarcoma. Cancer Causes Control. 2011;22:899-908.

27. Katz J, Lee ACC, Kozuki N, Lawn JE, Cousens S, Blencowe H, et al. Mortality risk in preterm and small-for-gestational-age infants in lowincome and middle-income countries: a pooled country analysis. Lancet. 2013;382:417-25.

28. Lau J, loannidis JP, Schmid CH. Quantitative synthesis in systematic reviews. Ann Intern Med. 1997;127:820-6.

29. DerSimonian R, Laird N. Meta-analysis in clinical trials. Control Clin Trials. 1986;7:177-88

30. Higgins JPT, Thompson SG, Spiegelhalter DJ. A re-evaluation of randomeffects meta-analysis. J R Stat Soc Ser A Stat Soc. 2009;172:137-59.

31. Higgins JPT. Heterogeneity in meta-analysis should be expected and appropriately quantified. Int J Epidemiol. 2008;37:1158-60.

32. Chinn S. A simple method for converting an odds ratio to effect size for use in meta-analysis. Stat Med. 2000;19:3127-31.

33. Cochran WG. The combination of estimates from different experiments. Biometrics. 1954;10:101.

34. Higgins JPT, Thompson SG. Quantifying heterogeneity in a meta-analysis. Stat Med. 2002;21:1539-58.

35. Sterne JAC, Sutton AJ, Ioannidis JPA, Terrin N, Jones DR, Lau J, et al. Recommendations for examining and interpreting funnel plot asymmetry in meta-analyses of randomised controlled trials. BMJ. 2011;343:d4002.
36. Egger M, Davey Smith G, Schneider M, Minder C. Bias in meta-analysis detected by a simple, graphical test. BMJ. 1997;315:629-34.

37. Ioannidis JPA, Trikalinos TA. An exploratory test for an excess of significant findings. Clin Trials. 2007:4:245-53.

38. Ioannidis JPA. Clarifications on the application and interpretation of the test for excess significance and its extensions. J Math Psychol. 2013;57:184-7.

39. Lubin JH, Gail MH. On power and sample size for studying features of the relative odds of disease. Am J Epidemiol. 1990;131:552-66.

40. Christian P, Lee SE, Donahue Angel M, Adair LS, Arifeen SE, Ashorn P, et al. Risk of childhood undernutrition related to small-for-gestational age and preterm birth in low- and middle-income countries. Int J Epidemiol. 2013;42:1340-55.

41. Mu M, Ye S, Bai M-J, Liu G-L, Tong Y, Wang S-F, et al. Birth weight and subsequent risk of asthma: a systematic review and meta-analysis. Hear Lung Circ. 2014;23:511-9.

42. Risnes KR, Vatten LJ, Baker JL, Jameson K, Sovio U, Kajantie E, et al. Birthweight and mortality in adulthood: a systematic review and metaanalysis. Int J Epidemiol. 2011;40:647-61.

43. Schellong K, Schulz S, Harder T, Plagemann A. Birth weight and longterm overweight risk: systematic review and a meta-analysis including 643,902 persons from 66 studies and 26 countries globally. PLoS One. 2012; $7:$ :47776.

44. Lau J, loannidis JPA, Terrin N, Schmid CH, Olkin I. The case of the misleading funnel plot. BMJ Br Med J. 2006;333:597-600.

45. Lawlor DA, Davey Smith G, Ebrahim S. Birth weight of offspring and insulin resistance in late adulthood: cross sectional survey. BMJ. 2002;325:359.

46. Berhan $Y$, Berhan A. A meta-analysis of selected maternal and fetal factors for perinatal mortality. Ethiop J Health Sci. 2014;24:55-68.

47. Knippenberg R, Lawn JE, Darmstadt GL, Begkoyian G, Fogstad H, Walelign $\mathrm{N}$, et al. Systematic scaling up of neonatal care in countries. Lancet. 2005;365:1087-98.

48. Baird J, Kurshid MA, Kim M, Harvey N, Dennison E, Cooper C. Does birthweight predict bone mass in adulthood? A systematic review and meta-analysis. Osteoporos Int. 2011;22:1323-34.

49. Wardlaw T, Blanc A, Zupan J, Ahman E. United Nations Children's Fund and World Health Organization, Low Birthweight: Country, Regional and Global Estimates. New York: UNICEF; 2004.

50. Tyrrell J, Richmond RC, Palmer TM, Feenstra B, Rangarajan J, Metrustry S, et al. Genetic evidence for causal relationships between maternal obesityrelated traits and birth weight. JAMA. 2016:315:1129.

51. HAPO Study Cooperative Research Group, Metzger BE, Lowe LP, Dyer AR, Trimble ER, Chaovarindr U, et al. Hyperglycemia and adverse pregnancy outcomes. N Engl J Med. 2008;358:1991-2002.

52. Chiswick C, Reynolds RM, Denison F, Drake AJ, Forbes S, Newby DE, et al. Effect of metformin on maternal and fetal outcomes in obese pregnant women (EMPOWaR): a randomised, double-blind, placebo-controlled trial. Lancet Diab Endocrinol. 2015;3:778-86.

53. Poston $L$, Bell R, Croker H, Flynn AC, Godfrey KM, Goff L, et al. Effect of a behavioural intervention in obese pregnant women (the UPBEAT study): a multicentre, randomised controlled trial. Lancet Diab Endocrinol. 2015;3:767-77.

54. Dodd JM, Turnbull D, MCPhee AJ, Deussen AR, Grivell RM, Yelland LN, et al. Antenatal lifestyle advice for women who are overweight or obese: LIMIT randomised trial. BMJ. 2014;348:g1285.

55. Loret de Mola C, de Franca GV A, Quevedo LDA, Horta BL. Low birth weight, preterm birth and small for gestational age association with adult depression: systematic review and meta-analysis. Br J Psychiatry. 2014;205:340-7.

56. Milne E, Greenop KR, Metayer C, Schüz J, Petridou E, Pombo-de-Oliveira MS, et al. Fetal growth and childhood acute lymphoblastic leukemia: findings from the childhood leukemia international consortium. Int J Cancer. 2013; 133:2968-79.

57. Delbaere I, Vansteelandt S, De Bacquer D, Verstraelen H, Gerris J, De Sutter $P$, et al. Should we adjust for gestational age when analysing birth weights? The use of z-scores revisited. Hum Reprod. 2007;22:2080-3.

58. Cook MB, Akre O, Forman D, Madigan MP, Richiardi L, McGlynn KA. A systematic review and meta-analysis of perinatal variables in relation to the risk of testicular cancer-experiences of the son. Int J Epidemiol. 2010;39:1605-18.

59. Araújo de França GV, Restrepo-Méndez MC, Loret de Mola C, Victora CG. Size at birth and abdominal adiposity in adults: a systematic review and meta-analysis. Obes Rev. 2014;15:77-91. 
60. Chen S, Yang L, Pu F, Lin H, Wang B, Liu J, et al. High birth weight increases the risk for bone tumor: A systematic review and meta-analysis. Int Environ Res Public Health. 2015;12:11178-95.

61. Davey Smith G, Hypponen E, Power C, Lawlor DA. Offspring birth weight and parental mortality: prospective observational study and meta-analysis. Am J Epidemiol. 2007:166:160-9.

62. Dodds R, Denison HJ, Ntani G, Cooper R, Cooper C, Sayer AA, et al. Birth weight and muscle strength: a systematic review and meta-analysis. J Nutr Health Aging. 2012;16:609-15.

63. Harder T, Plagemann A, Harder A. Birth weight and subsequent risk of childhood primary brain tumors: a meta-analysis. Am J Epidemiol. 2008;168:366-73.

64. Harder T, Roepke K, Diller N, Stechling Y, Dudenhausen JW, Plagemann A. Birth weight, early weight gain, and subsequent risk of type 1 diabetes: systematic review and meta-analysis. Am J Epidemiol. 2009;169:1428-36.

65. Harder T, Plagemann A, Harder A. Birth weight and risk of neuroblastoma: a meta-analysis. Int J Epidemiol. 2010;39:746-56.

66. Jackson S, Mathews KH, Pulanic D, Falconer R, Rudan I, Campbell H, et al. Risk factors for severe acute lower respiratory infections in children: a systematic review and meta-analysis. Croat Med J. 2013;54:110-21.

67. Kormos CE, Wilkinson AJ, Davey CJ, Cunningham AJ. Low birth weight and intelligence in adolescence and early adulthood: a meta-analysis. J Public Health (Oxf). 2014;36:213-24.

68. Lawlor DA, Ebrahim S, Davey SG. Association of birth weight with adult lung function: findings from the British Women's Heart and Health Study and a meta-analysis. Thorax. 2005:60:851-8.

69. Lawlor DA, Owen CG, Davies AA, Whincup PH, Ebrahim S, Cook DG, et al. Sex differences in the association between birth weight and total cholesterol. A meta-analysis. Ann Epidemiol. 2006;16:19-25.

70. Michos A, Xue F, Michels KB. Birth weight and the risk of testicular cancer: a meta-analysis. Int J Cancer. 2007;121:1123-31.

71. Mu M, Wang S-F, Sheng J, Zhao Y, Li H-Z, Hu C-L, et al. Birth weight and subsequent blood pressure: a meta-analysis. Arch Cardiovasc Dis. 2012; 105:99-113.

72. Øglund GP, Hildebrand M, Ekelund U. Are birth weight, early growth and motor development determinants of physical activity in children and youth? A systematic review and meta-analysis. Pediatr Exerc Sci. 2015;27(4):441-53.

73. Papadopoulou C, Antonopoulos CN, Sergentanis TN, Panagopoulou P, Belechri M, Petridou ET. Is birth weight associated with childhood lymphoma? A meta-analysis. Int J Cancer. 2012;130:179-89.

74. Panduru M, Salavastru CM, Panduru NM, Tiplica GS. Birth weight and atopic dermatitis: systematic review and meta-analysis. Acta Dermatovenerol Croat. 2014;22:91-6.

75. Silveira VMFD, Horta BL. Birth weight and metabolic syndrome in adults: meta-analysis. Rev Saude Publica. 2008;42:10-8.

76. Shi T, Balsells E, Wastnedge E, Singleton R, Rasmussen ZA, Zar HJ, et al. Risk factors for respiratory syncytial virus associated with acute lower respiratory infection in children under five years: systematic review and meta-analysis. J Glob Health. 2015;5:020416.

77. van Montfoort N, Finken MJJ, le Cessie S, Dekker FW, Wit JM. Could cortisol explain the association between birth weight and cardiovascular disease in later life? A meta-analysis Eur J Endocrinol. 2005;153:811-7.

78. Whincup P, Kaye S, Owen CG, Al E. Birth weight and risk of type 2 diabetes. JAMA. 2008;300:2886.

79. White SL, Perkovic V, Cass A, Chang CL, Poulter NR, Spector T, et al. Is low birth weight an antecedent of CKD in later life? A systematic review of observational studies. Am J Kidney Dis. 2009;54:248-61.

80. Xu X, Dailey AB, Peoples-Sheps M, Talbott EO, Li N, Roth J. Birth weight as a risk factor for breast cancer: a meta-analysis of 18 epidemiological studies. J Womens Health (Larchmt). 2009;18:1169-78.

81. Zhang Y, Li H, Liu S-J, Fu G-J, Zhao Y, Xie Y-J, et al. The associations of high birth weight with blood pressure and hypertension in later life: a systematic review and meta-analysis. Hypertens Res. 2013;36(8):725-35.

82. Hjalgrim LL, Westergaard T, Rostgaard K, Schmiegelow K, Melbye M, Hjalgrim $\mathrm{H}$, et al. Birth weight as a risk factor for childhood leukemia: a meta-analysis of 18 epidemiologic studies. Am J Epidemiol. 2003;158:724-35.

83. Xu X-F, Li Y-J, Sheng Y-J, Liu J-L, Tang L-F, Chen Z-M. Effect of low birth weight on childhood asthma: a meta-analysis. BMC Pediatr. 2014;14:275.

84. Flaherman V, Rutherford GW. A meta-analysis of the effect of high weight on asthma. Arch Dis Child. 2006:91:334-9.
85. Martínez-Mesa J, Restrepo-Méndez MC, González DA, Wehrmeister FC, Horta $\mathrm{BL}$, Domingues MR, et al. Life-course evidence of birth weight effects on bone mass: systematic review and meta-analysis. Osteoporos Int. 2012;24:7-18.

86. Zhao Y, Wang SF, Mu M, Sheng J. Birth weight and overweight/obesity in adults: a meta-analysis. Eur J Pediatr. 2012;171:1737-46.

87. Huxley R, Owen CG, Whincup PH, Cook DG, Rich-Edwards J, Smith GD, et al. Is birth weight a risk factor for ischemic heart disease in later life? Am J Clin Nutr. 2007;85:1244-50.

88. Yu ZB, Han SP, Zhu GZ, Zhu C, Wang XJ, Cao XG, et al. Birth weight and subsequent risk of obesity: a systematic review and meta-analysis. Obes Rev. 2011:12:525-42.

89. Huxley R, Owen CG, Whincup PH, Cook DG, Colman S, Collins R. Birth weight and subsequent cholesterol levels: exploration of the "fetal origins" hypothesis. JAMA. 2004;292:2755-64.

90. Owen CG, Whincup PH, Odoki K, Gilg JA, Cook DG. Birth weight and blood cholesterol level: a study in adolescents and systematic review. Pediatrics. 2003;111:1081-9.

\section{Submit your next manuscript to BioMed Central and we will help you at every step:}

- We accept pre-submission inquiries

- Our selector tool helps you to find the most relevant journal

- We provide round the clock customer support

- Convenient online submission

- Thorough peer review

- Inclusion in PubMed and all major indexing services

- Maximum visibility for your research

Submit your manuscript at www.biomedcentral.com/submit
C Biomed Central 